\title{
Microestructura cuticular del pelo de guardia en murciélagos del desierto costero del suroeste de Perú
}

\author{
Cuticular microstructure of the guard hair in bats from the coastal \\ desert of southern Peru
}

\author{
Yasmy K. Medina ${ }^{1}$, César E. Medina ${ }^{1,2}$, Evaristo López ${ }^{1}$
}

\section{Resumien}

\begin{abstract}
El presente estudio tuvo como objetivo describir la microestructura cuticular de los pelos de guardia de los murciélagos presentes en el desierto costero del sur de Perú. Se examinaron muestras de 45 especímenes de murciélagos (16 especies, 13 géneros y 4 familias), depositados en la colección científica de mamíferos del Museo de Historia Natural de la Universidad Nacional de San Agustín de Arequipa (MUSA). Se obtuvieron pelos de la zona inter-escapular, los cuales fueron observados en un Microscopio Electrónico de Barrido para obtener datos morfológicos y morfométricos. Se registraron cinco tipos de patrones cuticulares coronales (adpreso, divergente, divaricado, alternado y hastada asimétrica) y seis configuraciones del borde de las escamas (simple, hendido, emarginado, aserrado, denticulado y dentado), permitiendo diferenciar fácilmente los especímenes hasta el nivel de subfamilia, mientras que con ayuda de datos cuantitativos (largo del pelo, ancho del pelo, índice de escama e índice de ancho de escama) fue posible alcanzar una determinación hasta el nivel de especie. Se documenta, por primera vez, una descripción de la cutícula de pelos de guardia para 15 especies de murciélagos que habitan el desierto costero del sur de Perú. Se presenta una clave taxonómica que servirá de apoyo para el desarrollo de inventarios y programas de monitoreo y conservación de murciélagos en las costas del sur de Perú.
\end{abstract}

Palabras clave: clave taxonómica, escamas, especies amenazadas, inventarios biológicos, patrón cuticular

\footnotetext{
${ }^{1}$ Universidad Nacional de San Agustín de Arequipa, Arequipa, Perú

${ }^{2}$ E-mail: cmedinap@unsa.edu.pe; https://orcid.org/0000-0002-1275-223X
}

Recibido:10 de junio de 2020

Aceptado para publicación: 27 de febrero de 2021

Publicado: 23 de junio de 2021

(CLos autores. Este artículo es publicado por la Rev Inv Vet Perú de la Facultad de Medicina Veterinaria, Universidad Nacional Mayor de San Marcos. Este es un artículo de acceso abierto, distribuido bajo los términos de la licencia Creative Commons Atribución 4.0 Internacional (CC BY 4.0) [https:// creativecommons.org/licenses/by/4.0/deed.es] que permite el uso, distribución y reproducción en cualquier medio, siempre que la obra original sea debidamente citada de su fuente original 
The present study aimed to describe the cuticular microstructure of the guard hairs of bats present in the coastal desert of southern Peru. Samples of 45 bat specimens (16 species, 13 genera and 4 families) from the department of Arequipa were examined, which were part of the scientific collection of mammals of the Natural History Museum of the National University of San Agustín de Arequipa (MUSA). Hairs were obtained from the inter-scapular area, which were observed in a Scanning Electron Microscope to obtain morphological and morphometric data. Five types of coronal cuticular patterns (appressed, divergent, divaricated, alternate and unequal hastate) and six configurations of the edge of the scales (simple, cleft, emarginate, serrated, denticulate and dentate) were recorded, allowing the specimens to be differentiated down to the level of subfamily, while with the help of quantitative data (length and width of hair, Scale Index and Scale Width Index) it was possible to reach a determination down to the species level. For the first time, a description of the guard hair cuticle is documented for 15 species of bats that inhabit the coastal desert of southern Peru. A taxonomic key is presented that will support the development of inventories and monitoring and conservation programmes of bats on the southern coasts of Peru.

Key words: taxonomic key, scale, threatened species, biological inventories, cuticular pattern

\section{INTRODUCCIÓN}

La Tricología es una ciencia que emplea métodos no invasivos para la identificación de los mamíferos en base al análisis de la morfología del pelo, mediante la combinación de las principales características de la medula, corteza y cutícula (Quadros y Monteiro-Filho, 1998).

Teerink (1991) clasifica a los pelos en dos clases: (1) Pelos de cobertura o «underhair», los cuales funcionan como una capa que atrapa aire y aísla al animal de la intemperie. Dichos pelos son poco pigmentados, numerosos, ondulados y con constricciones. Se encuentran tres tipos de pelos de cobertura: lana (pelos largos, suaves y rizados), pelaje (pelos cortos, finos y abundantes) y vello (pelosfinos y cortos) (Martin et al., 2001); y (2) Pelos de guardia o «guard hair», los cuales ayudan a definir patrones de coloración, protegen los pelos de cobertura y repelen el agua. Estos pelos son largos, gruesos, fuer- temente pigmentados y sobresalen por encima del resto de pelos (Teerink, 1991; Fernández y Rossi 1998; Monroy-Vilchis et al., 2005). El pelo sufre notables modificaciones en algunos grupos de mamíferos, pudiendo constituirse como cerdas, espinas o placas (Cabrera, 1922), mientras que en la gran mayoría de grupos se presenta un pelo suave al tacto y sin mayores cambios estructurales macroscópicos y/o microscópicos. No obstante, el pelo de los murciélagos no presenta médula, característica única dentro del grupo de los mamíferos (Sessions et al., 2014).

El estudio de pelos en murciélagos se remonta a los mediados del Siglo XX, donde se describe y utiliza a los pelos como potenciales caracteres taxonómicos para distinguir entre especies de Norte América (Mathiak, 1938; Nason, 1948; Benedict, 1957). Posteriormente, la iniciativa se aplicó en algunas familias de murciélagos utilizando nuevos enfoques metodológicos en Sudamérica (Van Staaden y Jones, 1997), Centro América 
(Baca y Sánchez-Cordero, 2004; Schaetz et al., 2009), Norte América (Moore y Braun, 1983; Amman et al., 2002; Debelica y Thies, 2009) e India (Kumar et al., 2014).

Si bien, algunos autores consideran que la estructura del pelo posee un valor taxonómico limitado en murciélagos (Nason, 1948; Benedict, 1957), otros los han utilizado ampliamente para construir claves taxonómicas en base a los patrones cuticulares de las escamas, forma de la célula cortical y patrones de pigmentación (Mathiak, 1938; Moore y Braun, 1983). Por tanto, considerando el potencial de la microestructura del pelo como carácter diagnóstico y la escasa información sobre pelos de murciélagos en Sudamérica (Benedict, 1957; Van Staaden y Jones, 1997), el presente estudio tuvo como objetivo describir la microestructura cuticular de los pelos de guardia de los murciélagos presentes en el desierto costero del sur de Perú.

\section{Materiales y Métodos}

Quadros y Monteiro-Filho (1998) observaron que los procedimientos de taxidermia no dañan o alteran los patrones medulares y cuticulares de los pelos, lo cual permitió utilizar pelos de colecciones científicas de $\mathrm{Mu}$ seos de Historia Natural en este estudio como material de referencia para la identificación de las especies de mamíferos que se encuentran en egagrópilas, excrementos, etc. Por consiguiente, se examinaron pelos de 16 de las 18 especies de murciélagos reportadas para el Departamento de Arequipa, Perú (Pari et al., 2015; Medina et al., 2018; Málaga et al., 2020), los cuales constituyen una muestra representativa del ensamble de murciélagos que ocurre en el Desierto Costero del sur de Perú (Cuadro 1).

Los pelos fueron obtenidos de 45 especímenes depositados en la colección científica de mamíferos del Museo de Historia Natural de la Universidad Nacional de San Agustín de Arequipa (MUSA), los cuales se agruparon taxonómicamente en 16 especies, 13 géneros y 4 familias. Tres individuos por especie fueron seleccionados al azar para colectar 10 muestras de pelo de la zona interescapular de cada murciélago, siguiendo la metodología de Arita y Aranda (1987) y Quadros y Monteiro-Filho (2006). Los pelos fueron limpiados con solución jabonosa y alcohol al 70\%, según Kshirsagar et al. (2009) y Fasola et al. (2005), procesándose de 20 a 30 pelos por especie.

Los pelos de los murciélagos se clasifican en tres tipos: lanosos, primarios y secundarios, en los cuales se distinguen tres regiones: la raíz, que incluye el bulbo del pelo; el tallo, que constituye la mayor longitud del pelo; y la espátula, apéndice típico con forma de lanza (Pierallini et al., 2004). Por tanto, la descripción macroscópica fue realizada en base a la coloración, número de bandas (bandeo) y largo de los pelos (LP), mientras que para la descripción microscópica se utilizó la parte distal o espátula del pelo de guardia secundario, siguiendo las definiciones y esquemas reportadas en la literatura científica (Nason, 1948; Benedict, 1957; Arita y Aranda, 1987; Amman et al., 2002; Pierallini et al., 2004; Schaetz et al., 2009).

Se obtuvieron microfotografías de la cutícula, parte distal de los pelos, con ayuda de un Microscopio Electrónico de Barrido (MEB) FEI Inspect TM S50, conectado al programa xT microscope Control. Se trabajó con una magnificación de $2600 \mathrm{x}$, SPOT $=$ $4-5, \mathrm{HV}=3.5-10 \mathrm{kV}$, Presión $=1.06 \mathrm{E}-06-$ 2.01E-05 Torr, $\mathrm{WD}=9.6-11.4 \mathrm{~mm}, \mathrm{HFW}=$ $115 \mu \mathrm{m}$.

Se registraron las siguientes medidas: Largo de escama (LE), Ancho máximo de escama (AMaxE) y Ancho mínimo de escama (AMinE), las cuales permitieron estimar el Índice de Escama (IS = LE/AMaxE) y el Índice de Ancho de Escama (WI = AMaxE/ AMinE) (Debelica y Thies, 2009). Análisis de componentes principales (ACP) fueron lle- 
Cuadro 1. Especímenes de murciélagos del desierto costero del sur de Perú examinados para determinar las características del pelo de guardia (Museo de Historia Natural de la Universidad Nacional de San Agustín de Arequipa

\begin{tabular}{|c|c|c|}
\hline $\begin{array}{l}\text { Familia/Subfamilia/ } \\
\text { Especie }\end{array}$ & Localidad & $\begin{array}{l}\text { N. }{ }^{\circ} \text { de catálogo } \\
\text { MUSA }\end{array}$ \\
\hline \multicolumn{3}{|l|}{ Phyllostomidae } \\
\hline \multicolumn{3}{|l|}{ Desmodontinae } \\
\hline \multicolumn{3}{|c|}{ 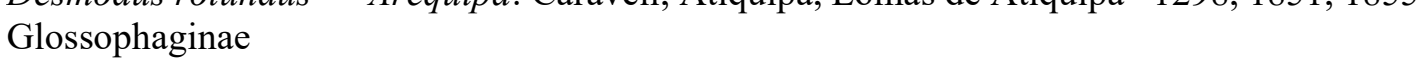 } \\
\hline Glossophaga valens & Arequipa: Condesuyos, Yanatile, La Barrera & $16984,16979,16981$ \\
\hline \multirow{3}{*}{ Platalina genovensium } & Arequipa: Arequipa, Tiabaya, quebrada Linga & 239 \\
\hline & Arequipa: Castilla, Ayo, laguna Mamacocha & 2712 \\
\hline & Arequipa: Arequipa, Yarabamba, Mina El Rescate & 6425 \\
\hline \multicolumn{3}{|l|}{ Stenodermatinae } \\
\hline \multirow[t]{3}{*}{ Sturnira bogotensis } & Lima: Yauyos, Alis & 5373 \\
\hline & Cajamarca: Cajamarca, San Juan, Sunchubamba & 11228 \\
\hline & Arequipa: Castilla, Ayo, laguna Mamacocha & 2718 \\
\hline \multicolumn{3}{|c|}{ 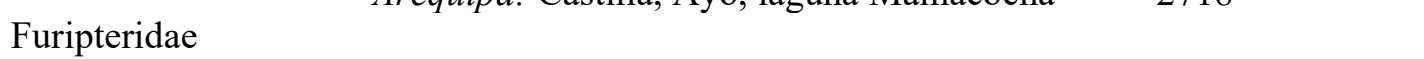 } \\
\hline Amorphochilus & Arequipa: Caravelí, Atiquipa, Cerro Fronton & 1305,1306 \\
\hline \multicolumn{3}{|l|}{ Molossidae } \\
\hline \multicolumn{3}{|l|}{ Tomopeatinae } \\
\hline \multirow[t]{3}{*}{ Tomopeas ravus } & Arequipa: Condesuyos, Yanaquihua, Ispacas & 14392 \\
\hline & Arequipa: La Unión, Toro, Puglle & 14500 \\
\hline & Piura, Talara, Lobitos, quebrada Los Amarillos & 14256 \\
\hline \multicolumn{3}{|c|}{ 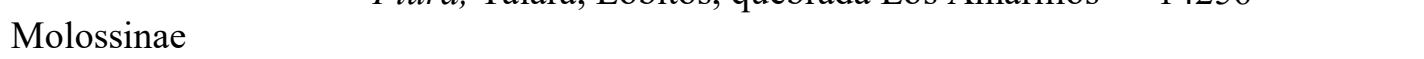 } \\
\hline Molossus molossus & Puno: Sandia, San Juan del Oro & $9603,9686,9687$ \\
\hline Mormopterus & Arequipa: Arequipa, Vitor, Sotillo & 13600,13601 \\
\hline kalinowskii & & 13605 \\
\hline $\begin{array}{l}\text { Nyctinomops } \\
\text { aurispinosus }\end{array}$ & Arequipa: Camaná & $\begin{array}{l}2659 \\
2660\end{array}$ \\
\hline Nyctinomops & Arequipa: Caravelí, Atiquipa, Lomas de Atiquipa, & 1295 \\
\hline 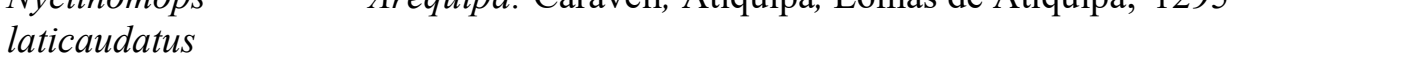 & Cusco: Urubamba, Machupicchu, Rio Aobamba & 15837 \\
\hline \multirow[t]{2}{*}{ Nyctinomops macrotis } & Arequipa: Arequipa, Paucarpata, Porongoche & 039 \\
\hline & Arequipa & 11326 \\
\hline \multirow[t]{2}{*}{ Promops davisoni } & Arequipa: Condesuyos, Iquipí, Rio Grande & 16970 \\
\hline & Condesuyos, Yanatile, La Barrera & 16980,16983 \\
\hline Tadarida brasiliensis & Arequipa: Camaná, Camaná & $13430,13425,13429$ \\
\hline \multicolumn{3}{|c|}{ Vespertilionidae } \\
\hline \multicolumn{3}{|l|}{ Vespertilioninae } \\
\hline $\begin{array}{l}\text { Eptesicus montanus } \\
\text { Myotinae }\end{array}$ & Arequipa: Condesuyos, Iquipí, Rio Grande & $16963,16964,16972$ \\
\hline \multirow[t]{3}{*}{ Myotis atacamensis } & Arequipa: Arequipa & 1860 \\
\hline & Arequipa: Arequipa, La Joya, San Isidro & 2641 \\
\hline & Arequipa: Condesuyos, Iquipí & 9820 \\
\hline Myotis cf oxyotus & Arequipa: Condesuyos, Iquipí & $9814,9815,9826$ \\
\hline
\end{tabular}


vados a cabo usando una matriz de varianzacovarianza en base a las cuatro medidas tomadas, las cuales fueron normalizadas usando la transformación logarítmica. Los primeros tres componentes fueron obtenidos y los pesos de todas las variables fueron evaluados como una medida de importancia en esta separación preliminar. Las diferencias estadísticas entre grupos fueron evaluadas usando análisis de función discriminante (AFD) (Brown y Wicker, 2000) y la validación cruzada fue evaluada usando la opción «leave out» en el programa PAST. Los análisis se realizaron con el software PAST 3.0 para Windows (Hammer, 2013).

\section{Resultados}

Se encontraron cinco tipos de patrones cuticulares coronales, los cuales permiten diferenciar a los especímenes a nivel de familia (Cuadro 2). Los murciélagos Phyllostomidae presentan los patrones cuticulares adpreso y alternado, los Furipteridae tienen un patrón divergente, los Molossidae presentan patrones divergente y divaricado y los Vespertilionidae es de tipo hastada asimétrico (Figuras 1, 2).

Así mismo, se reconocieron seis configuraciones del borde de las escamas, permitiendo diferenciar fácilmente los especímenes hasta el nivel de subfamilia de la siguiente manera:

- Desmodontinae (Desmodus rotundus): Escamas adpresas con bordes hendidos.

- Phyllostominae (Platalina genovensium) y Glossophaginae (Glossophaga valens): Escamas alternadas con bordes simples y emarginados.

- Sternodermatinae (Sturnira bogotensis): Escamas alternadas con bordes denticulados.

- Tomopeatinae (Tomopeas ravus): Escamas divaricadas con bordes denticulados.

- Molossinae (Molossus molossus, Nyctinomops macrotis y Promops davisoni) presentan escamas divergen- tes y bordes dentados. (Mormopterus kalinowskii, Nyctinomops aurispinosus, N. laticaudatus y Tadarida brasiliensis) presentan escamas divaricadas, ambas con bordes dentados.

Los Cuadros 2 y 3 detallan los caracteres macro y microscópicos de los pelos de guardia de cada una de las especies revisadas.

Con base a las medidas tomadas (Cuadro 3), los tres primeros componentes del ACP recuperaron el $95.12 \%$ de la variación total, observándose cierto grado de agrupación entre las especies que pertenecen a una misma familia o subfamilia (Figura 3). El componente principal $1(\mathrm{CP} 1)$ recuperó el mayor porcentaje de variación (43.22\%), siendo el Largo de escama el carácter que presentó el mayor valor positivo (Cuadro 4). El AFD mostró que 57.2 y $21.61 \%$ de la variación es explicado por el primer y segundo eje canónico (Cuadro 4). La matriz de factores discriminantes indica que el Largo de pelo tiene una alta correlación positiva con el axis de la primera función discriminante, mientras que el Ancho mínimo de escama con la segunda función discriminante. El AFD muestra una correcta asignación de los individuos a los grupos previamente definidos; sin embargo, utilizando la prueba de Jackknifed, solo $56.88 \%$ de los casos estarían correctamente asignados.

En base a la combinación de datos morfológicos y morfométricos se desarrolló una clave dicotómica para determinar las especies de murciélagos del Desierto costero en el sur de Perú (Cuadro 5).

\section{Discusión}

El pelo presenta resistencia a factores físicos, químicos, mecánicos y biológicos (Quadros y Monteiro-Filho, 1998), motivo por el cual es ampliamente utilizado en la industria textil, criminalística, ecología trófica y paleontología. No obstante, la falta de catálogos taxonómicos de pelos reduce la calidad de sus productos y aplicaciones. 
Cuadro 2. Matriz de característica externa y patrones cuticulares murciélagos en la región Arequipa (Museo de Historia Natural de la Universidad Nacional de San Agustín de Arequipa

\begin{tabular}{|c|c|c|c|c|c|}
\hline \multirow[b]{2}{*}{ Familia/Especie } & \multirow{2}{*}{$\begin{array}{c}\text { Externa } \\
\text { Bandas } \\
\text { de color }\end{array}$} & \multicolumn{4}{|c|}{ Patrones cuticulares } \\
\hline & & $\begin{array}{l}\text { Dimensión } \\
\text { de escama }\end{array}$ & $\begin{array}{c}\text { Ornamentación } \\
\text { de bordes de } \\
\text { escamas }\end{array}$ & $\begin{array}{c}\text { Forma de } \\
\text { escama } \\
\text { coronal } \\
\end{array}$ & $\begin{array}{c}\text { Borde de } \\
\text { escama } \\
\text { coronal } \\
\end{array}$ \\
\hline \multicolumn{6}{|l|}{ Phyllostomidae } \\
\hline $\begin{array}{l}\text { Desmodus } \\
\text { rotundus }\end{array}$ & $\mathrm{O}-\mathrm{C}$ & Anchas & Adornada & Divergente & Hendidos \\
\hline $\begin{array}{l}\text { Glossophaga } \\
\text { valens }\end{array}$ & $\mathrm{O}-\mathrm{C}$ & Estrecha & Lisa & Alternada & Emarginado \\
\hline $\begin{array}{l}\text { Platalina } \\
\text { genovensium }\end{array}$ & $\mathrm{O}-\mathrm{C}$ & Intermedia & Lisa & Alternada & Simple \\
\hline $\begin{array}{l}\text { Sturnira } \\
\text { bogotensis }\end{array}$ & $\mathrm{O}-\mathrm{C}-\mathrm{O}$ & Intermedia & Adornada & Alternada & Denticulados \\
\hline \multicolumn{6}{|l|}{ Furipteridae } \\
\hline $\begin{array}{l}\text { Amorphochilus } \\
\text { schnablii }\end{array}$ & $\mathrm{C}-\mathrm{O}$ & Anchas & Adornada & Divergente & Aserrado \\
\hline \multicolumn{6}{|l|}{ Molossidae } \\
\hline Tomopeas ravus & $\mathrm{C}-\mathrm{O}$ & Intermedia & Adornada & Divaricada & Denticulados \\
\hline $\begin{array}{l}\text { Molossus } \\
\text { molossus }\end{array}$ & $\mathrm{O}-\mathrm{C}$ & Anchas & Adornada & Divergente & Dentados \\
\hline $\begin{array}{l}\text { Mormopterus } \\
\text { kalinowskii }\end{array}$ & $\mathrm{O}-\mathrm{C}$ & Anchas & Adornada & Divaricada & Dentados \\
\hline N. aurispinosus & $\mathrm{O}-\mathrm{C}$ & Anchas & Adornada & Divaricada & Dentados \\
\hline N. laticaudatus & $\mathrm{O}-\mathrm{C}$ & Anchas & Adornada & Divaricada & Dentados \\
\hline N. macrotis & $\mathrm{O}-\mathrm{C}$ & Anchas & Adornada & Divergente & Dentados \\
\hline $\begin{array}{l}\text { Promops } \\
\text { davisoni }\end{array}$ & $\mathrm{O}-\mathrm{C}-\mathrm{C}$ & Anchas & Adornada & Divergente & Dentados \\
\hline $\begin{array}{l}\text { Tadarida } \\
\text { brasiliensis }\end{array}$ & $\mathrm{O}-\mathrm{C}$ & Anchas & Adornada & Divaricada & Dentados \\
\hline \multicolumn{6}{|l|}{ Vespertilionidae } \\
\hline $\begin{array}{l}\text { Eptesicus } \\
\text { montanus }\end{array}$ & $\mathrm{C}-\mathrm{O}$ & Anchas & Lisa & $\begin{array}{l}\text { Hastada } \\
\text { asimétrica }\end{array}$ & Simple \\
\hline $\begin{array}{l}\text { Myotis } \\
\text { atacamensis }\end{array}$ & $\mathrm{C}-\mathrm{O}$ & Estrecha & Lisa & $\begin{array}{l}\text { Hastada } \\
\text { asimétrica }\end{array}$ & Simple \\
\hline M. cf. oxyotus & $\mathrm{C}-\mathrm{O}$ & Estrecha & Lisa & $\begin{array}{l}\text { Hastada } \\
\text { asimétrica }\end{array}$ & Simple \\
\hline
\end{tabular}

Bandas de color: O: Oscuro y C: Claro 

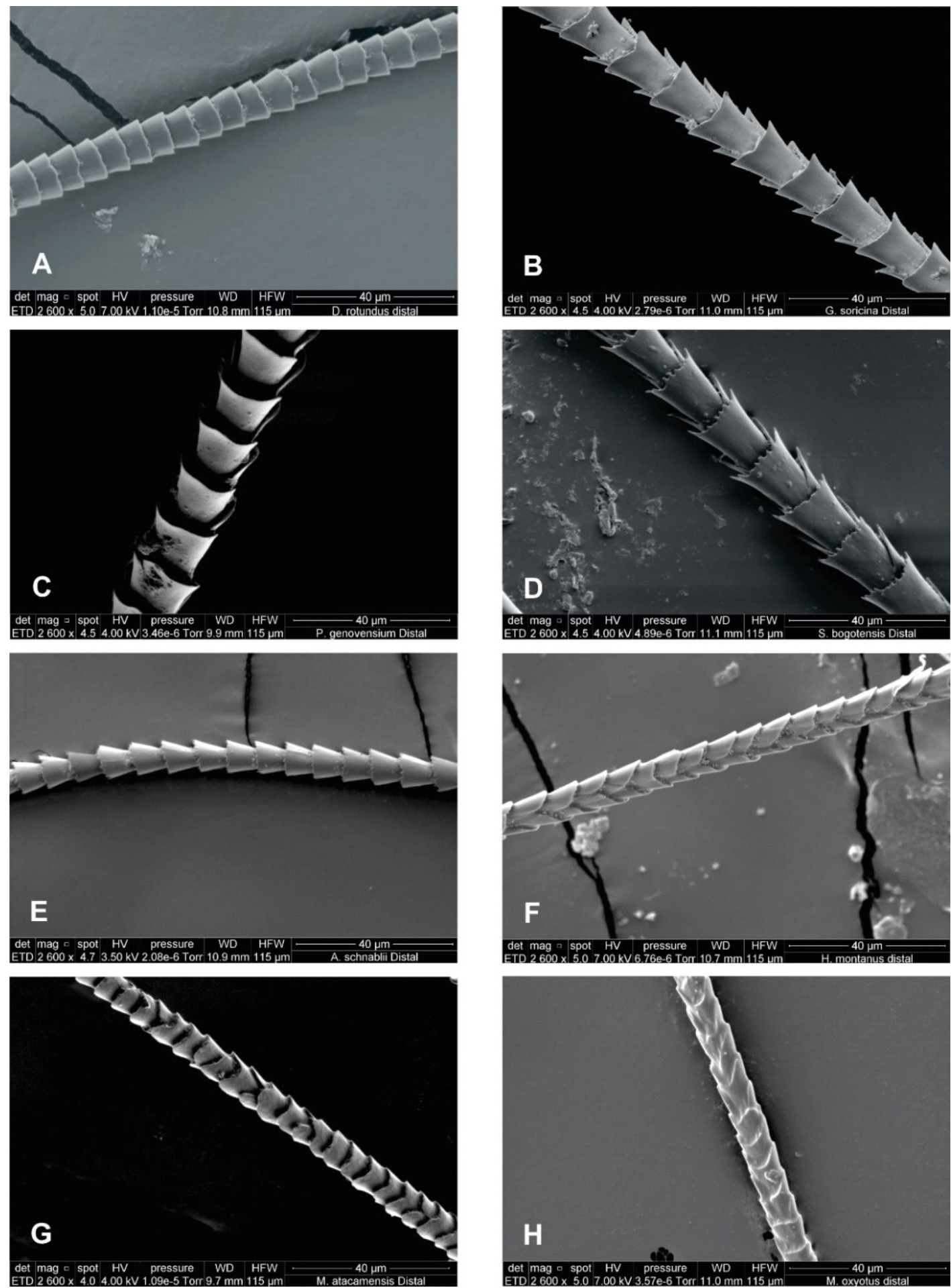

Figura 1. Patrones cuticulares de pelos de guardia de murciélagos de las familias Phyllostomidae, Furipteridae y Vespertilionidae presentes en la Región Arequipa, Perú: A, Desmodus rotundus (MUSA 1855); B, Glossophaga valens (MUSA 16979); C, Platalina genovensium (MUSA 6425); D, Sturnira bogotensis (MUSA 11228); E, Amorphochilus schnablii (MUSA 9386); F, Eptesicus montanus (MUSA 16964); G, Myotis atacamensis (MUSA 9820); H, Myotis cf. oxyotus (MUSA 9826) 

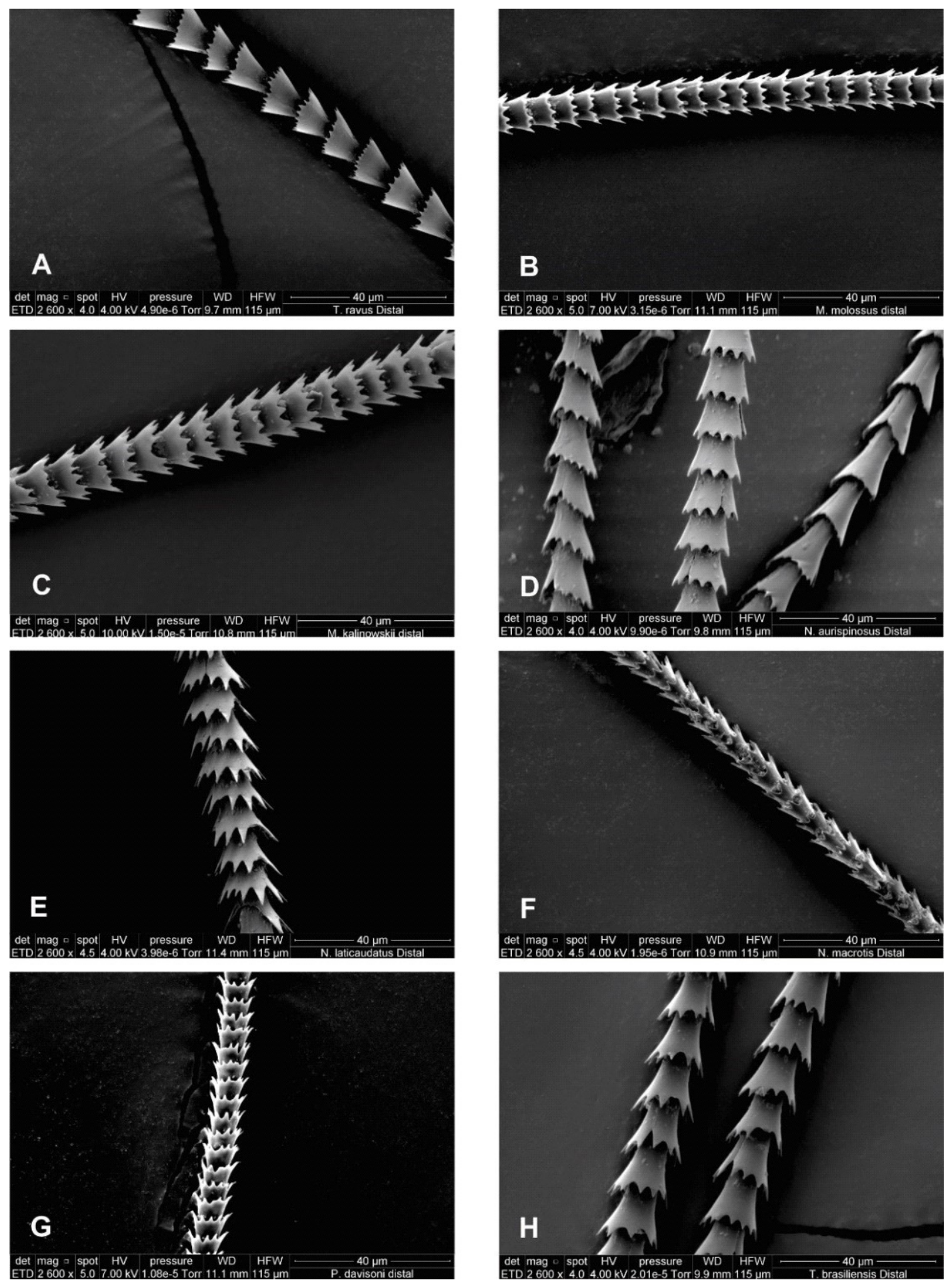

Figura 2. Patrones cuticulares de pelos de guardia de murciélagos de las familias Molossidae presentes en la Región Arequipa, Perú: A, Tomopeas ravus (MUSA 14500); B, Molossus molossus (MUSA 9687); C, Mormopterus kalinowskii (MUSA 13601); D, Nyctinomops aurispinosus (MUSA 2659); E, Nyctinomops laticaudatus (MUSA 1295); F, Nyctinomops macrotis (MUSA 11326); G, Promops davisoni (MUSA 16983); $\mathrm{H}$, Tadarida brasiliensis (MUSA 13429) 
Cuadro 3. Datos cuantitativos (promedio \pm d.e.) de los pelos de guardia de murciélagos en la Región Arequipa (Museo de Historia Natural de la Universidad Nacional de San Agustín de Arequipa)

\begin{tabular}{|c|c|c|c|c|c|c|}
\hline \multirow[b]{2}{*}{ Familia/Especie } & \multirow[b]{2}{*}{$\begin{array}{l}\text { Longitud } \\
\text { de pelo } \\
(\mathrm{mm})\end{array}$} & \multicolumn{5}{|c|}{ Escama } \\
\hline & & $\begin{array}{l}\text { Largo } \\
(\mu \mathrm{m})\end{array}$ & $\begin{array}{l}\text { Ancho } \\
\text { mínimo } \\
(\mu \mathrm{m})\end{array}$ & $\begin{array}{l}\text { Ancho } \\
\text { máximo } \\
(\mu \mathrm{m})\end{array}$ & $\begin{array}{l}\text { Índice } \\
\text { (IS) }\end{array}$ & $\begin{array}{l}\text { Índice } \\
\text { ancho } \\
(\mathrm{WI})\end{array}$ \\
\hline \multicolumn{7}{|l|}{ Phyllostomidae $^{1}$} \\
\hline Desmodus rotundus & $\begin{array}{c}10.83 \pm \\
1.83\end{array}$ & $\begin{array}{c}7.54 \pm \\
1.21\end{array}$ & $\begin{array}{c}7.52 \pm \\
1.19\end{array}$ & $\begin{array}{c}9.63 \pm \\
1.42\end{array}$ & 0.78 & 1.28 \\
\hline Glossophaga valens & $\begin{array}{c}6.17 \pm \\
0.82\end{array}$ & $\begin{array}{c}19.71 \pm \\
5.07\end{array}$ & $\begin{array}{l}9.15 \pm \\
2.99\end{array}$ & $\begin{array}{c}13.93 \pm \\
3.73\end{array}$ & 1.41 & 1.52 \\
\hline Platalina genovensium & $\begin{array}{c}9.00 \pm \\
1.41\end{array}$ & $\begin{array}{c}17.52 \pm \\
4.40\end{array}$ & $\begin{array}{c}12.45 \pm \\
1.10\end{array}$ & $\begin{array}{c}17.25 \pm \\
1.66\end{array}$ & 1.02 & 1.39 \\
\hline Sturnira bogotensis & $\begin{array}{c}8.67 \pm \\
1.15\end{array}$ & $\begin{array}{c}18.59 \pm \\
6.59\end{array}$ & $\begin{array}{c}12.19 \pm \\
1.10\end{array}$ & $\begin{array}{c}17.17 \pm \\
2.00\end{array}$ & 1.08 & 1.41 \\
\hline $\begin{array}{l}\text { Furipteridae } \\
\text { Amorphochilus } \\
\text { schnablii }\end{array}$ & $\begin{array}{c}9.00 \pm \\
1.41\end{array}$ & $\begin{array}{c}10.29 \pm \\
2.26\end{array}$ & $\begin{array}{c}6.68 \pm \\
0.54\end{array}$ & $\begin{array}{c}12.34 \pm \\
0.90\end{array}$ & 0.83 & 1.85 \\
\hline \multicolumn{7}{|l|}{ Molossidae } \\
\hline Tomopeas ravus ${ }^{1}$ & $\begin{array}{c}7.50 \pm \\
1.29\end{array}$ & $\begin{array}{c}15.58 \pm \\
2.45\end{array}$ & $\begin{array}{c}4.58 \pm \\
0.93\end{array}$ & $\begin{array}{c}15.07 \pm \\
1.38\end{array}$ & 1.03 & 3.29 \\
\hline Molossus molossus ${ }^{1}$ & $\begin{array}{c}5.00 \pm \\
0.82\end{array}$ & $\begin{array}{c}9.04 \pm \\
1.53\end{array}$ & $\begin{array}{c}6.55 \pm \\
0.47\end{array}$ & $\begin{array}{c}11.17 \pm \\
0.82\end{array}$ & 0.81 & 1.71 \\
\hline $\begin{array}{l}\text { Mormopterus } \\
\text { kalinowskii }^{1}\end{array}$ & $\begin{array}{c}4.83 \pm \\
0.82\end{array}$ & $\begin{array}{c}11.52 \pm \\
1.44\end{array}$ & $\begin{array}{c}7.33 \pm \\
1.38\end{array}$ & $\begin{array}{c}16.78 \pm \\
1.56\end{array}$ & 0.69 & 2.29 \\
\hline $\begin{array}{l}\text { Nyctinomops } \\
\text { aurispinosus }{ }^{2}\end{array}$ & $\begin{array}{c}5.00 \pm \\
1.00\end{array}$ & $\begin{array}{c}14.10 \pm \\
2.08\end{array}$ & $\begin{array}{c}9.64 \pm \\
1.90\end{array}$ & $\begin{array}{c}19.63 \pm \\
3.02\end{array}$ & 0.72 & 2.04 \\
\hline $\begin{array}{l}\text { Nyctinomops } \\
\text { laticaudatus }^{2}\end{array}$ & $\begin{array}{c}4.75 \pm \\
1.58\end{array}$ & $\begin{array}{c}13.87 \pm \\
1.34\end{array}$ & $\begin{array}{c}9.43 \pm \\
1.16\end{array}$ & $\begin{array}{c}19.17 \pm \\
1.38\end{array}$ & 0.72 & 2.03 \\
\hline Nyctinomops macrotis ${ }^{2}$ & $\begin{array}{c}7.50 \pm \\
1.00\end{array}$ & $\begin{array}{c}10.61 \pm \\
1.16\end{array}$ & $\begin{array}{c}8.95 \pm \\
0.99\end{array}$ & $\begin{array}{c}15.15 \pm \\
1.08\end{array}$ & 0.70 & 1.69 \\
\hline Promops davisoni $^{1}$ & $\begin{array}{l}7.67 \pm \\
1.83\end{array}$ & $\begin{array}{c}9.51 \pm \\
1.68\end{array}$ & $\begin{array}{l}7.11 \pm \\
0.65\end{array}$ & $\begin{array}{c}12.34 \pm \\
1.44\end{array}$ & 0.77 & 1.74 \\
\hline Tadarida brasiliensis ${ }^{1}$ & $\begin{array}{c}7.00 \pm \\
1.40\end{array}$ & $\begin{array}{c}15.56 \pm \\
1.75\end{array}$ & $\begin{array}{c}8.78 \pm \\
1.48\end{array}$ & $\begin{array}{c}17.48 \pm \\
1.73\end{array}$ & 0.89 & 1.99 \\
\hline \multicolumn{7}{|l|}{ Vespertilionidae $^{1}$} \\
\hline Eptesicus montanus & $\begin{array}{c}9.67 \pm \\
2.52\end{array}$ & $\begin{array}{c}16.82 \pm \\
2.14\end{array}$ & $\begin{array}{l}8.31 \pm \\
1.43\end{array}$ & $\begin{array}{c}13.96 \pm \\
1.50\end{array}$ & 1.20 & 1.68 \\
\hline Myotis atacamensis & $\begin{array}{c}9.33 \pm \\
1.15\end{array}$ & $\begin{array}{c}16.22 \pm \\
2.33\end{array}$ & $\begin{array}{c}7.81 \pm \\
1.09\end{array}$ & $\begin{array}{c}12.92 \pm \\
0.96\end{array}$ & 1.26 & 1.65 \\
\hline Myotis cf. oxyotus & $\begin{array}{c}10.00 \pm \\
0.82\end{array}$ & $\begin{array}{c}17.39 \pm \\
3.66\end{array}$ & $\begin{array}{c}6.49 \pm \\
1.14\end{array}$ & $\begin{array}{c}10.73 \pm \\
1.78\end{array}$ & 1.62 & 1.65 \\
\hline
\end{tabular}

${ }^{1} 30$ pelos por especie; ${ }^{2} 20$ pelos por especie 
Cuadro 4. Factores extraídos del análisis de componentes principales y del análisis de función discriminante para 16 especies de murciélagos del desierto costero del sur de Perú (Museo de Historia Natural de la Universidad Nacional de San Agustín de Arequipa), en base a una matriz de varianzacovarianza

\begin{tabular}{lllllll}
\hline \multirow{2}{*}{ Caracteres } & \multicolumn{3}{l}{ Componentes principales } & \multicolumn{3}{l}{ Función Discriminante } \\
\cline { 2 - 7 } & PC1 & PC2 & PC3 & Axis 1 & Axis 2 & Axis 3 \\
\hline Largo de pelo & -0.1845 & 0.8059 & 0.4511 & 10.400 & 8.609 & -7.851 \\
Largo de la escama & 0.6584 & 0.5156 & -0.5182 & 0.915 & 5.977 & 6.434 \\
Ancho mínimo de escama & 0.5214 & -0.0974 & 0.7204 & 10.119 & 13.795 & 14.348 \\
Ancho máximo de escama & 0.5105 & -0.2741 & 0.0956 & -20.390 & -9.113 & -6.209 \\
Eigenvalue & 0.0277 & 0.0193 & 0.0139 & 9.692 & 3.662 & 2.704 \\
Varianza explicada (\%) & 43.216 & 30.139 & 21.768 & 57.20 & 21.61 & 15.96 \\
\hline
\end{tabular}

Información sobre la correcta identificación de los pelos de murciélagos en Sudamérica es limitada (Van Staaden y Jones, 1997). A pesar de que existen colecciones biológicas importantes de mamíferos en los museos de historia natural del Perú, sólo se cuenta con un estudio de pelos a nivel nacional (Medina et al., 2018), lo cual pone en evidencia la relevancia del presente estudio.

Los pelos observados en las cuatro familias de murciélagos estudiadas (Phyllostomidae, Furipteridae, Molossidae y Vespertilionidae) carecieron de médula, lo cual concuerda con lo observado por diversos autores (van Staaden y Jones, 1997; Baca y Sánchez-Cordero, 2004; Debelica y Thies, 2009); sin embargo, al realizar la descripción de los patrones coronales, y compararlos con descripciones previas, se observó una falta de uniformidad en las definiciones, como a continuación se detalla:

- Las especies de la familia Phyllostomidae, subfamilia Desmodontinae presentaron escamas coronales adpresas hendidas, mientras que las subfamilias
Glossophaginae y Sternodermatinae presentaron escamas alternadas simples y emarginadas. Benedict (1957) observó que $D$. rotundus tenía escamas de forma coronal corta, repanada a sinuosa con menores irregularidades marginales; sin embargo, Schaetz et al. (2009) lo describen como escamas divergentes hendidas. Respecto a las subfamilias Glossophaginae y Sternodermatinae, Baca y Sánchez-Cordero (2004) describen las escamas de G. soricina como coronal hastada asimétrica; mientras que, Schaetz et al. (2009) describen las escamas coronales como alternadas, corroborando las observaciones del presente estudio. En la familia Furipteridae, Amorphochilus schnablii presentó escamas coronales divergentes denticuladas; concordando con lo observado por Benedict (1957).

- En la familia Molossidae se encontraron escamas de tipo divergentes y divaricadas con bordes dentados. Benedict (1957) encontró escamas coronales dentadas con una simple y profunda incisión 

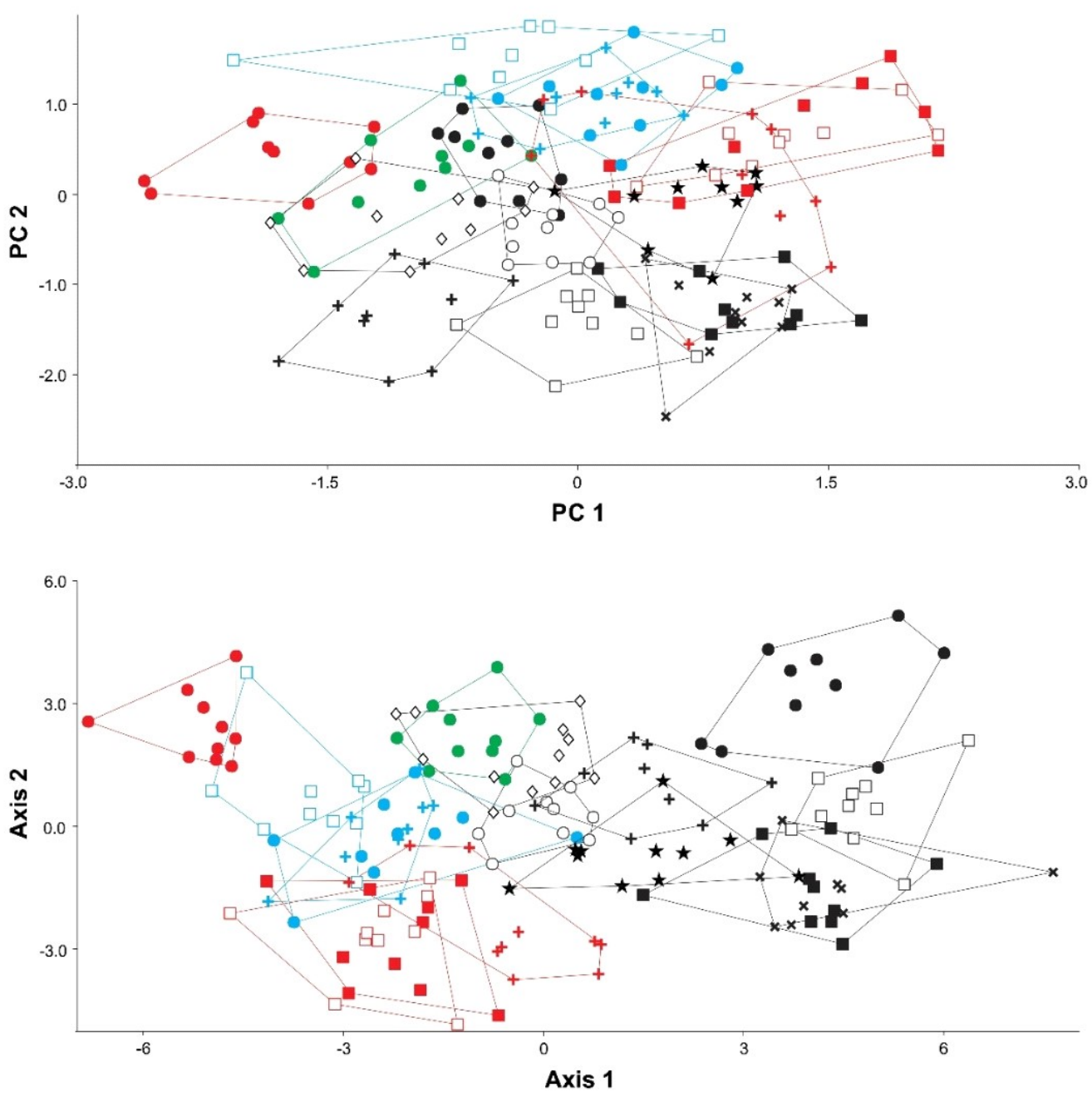

Figura 3. Resultados del análisis de componentes principales - ACP (arriba) y del análisis de función discriminante - AFD (abajo) de pelos de guardia de murciélagos (n=16), extraído desde cinco medidas. En color rojo, familia Phyllostomidae (Desmodus rotundus, círculos llenos; Glossophaga valens, cruces; Platalina genovensium, cuadrados vacíos; Sturnira bogotensis, cuadrados llenos). En color verde, familia Furipteridae (Amorphochilus schnablii). En color negro, familia Molossidae (Tomopeas ravus, círculos 1lenos; Molossus molossus, cruces; Mormopterus kalinowskii, cuadrados vacíos; Nyctinomops aurispinosus, cuadrados llenos; Nyctinomops laticaudatus, equis; Nyctinomops macrotis, círculo vacío; Promops davisoni, diamante vacío; Tadarida brasiliensis, estrellas). En color celeste, familia Vespertilionidae (Eptesicus montanus, círculos llenos; Myotis atacamensis, cruces; Myotis cf oxyotus, cuadrados vacíos) 
Cuadro 5. Clave dicotómica para determinar las especies de murciélagos del Desierto costero del sur de Perú

\begin{tabular}{|c|c|c|}
\hline $\mathrm{N}^{\circ}$ & Característica & Especie \\
\hline 1 & $\begin{array}{l}\text { Escama coronal hastada, con bordes asimétricos } \\
\text { (Vespertilionidae) }\end{array}$ & 2 \\
\hline $1^{\prime}$ & Escama coronal adpresa, divergente, divaricada o alternada & 4 \\
\hline 2 & Índice de la escama (IS) $>1.44$ & Myotis cf. Oxyotus \\
\hline $2^{\prime}$ & Índice de la escama (IS) $<1.45$ & 3 \\
\hline 3 & Escamas estrechas $(\mathrm{AminE}<8.00 \mu \mathrm{m} ; \mathrm{AmaxE}<13.43 \mu \mathrm{m})$ & Myotis atacamensis \\
\hline $3^{\prime}$ & Escamas anchas $($ AminE $>8.05 \mu \mathrm{m} ;$ AmaxE $>13.44 \mu \mathrm{m})$ & Eptesicus montanus \\
\hline 4 & $\begin{array}{l}\text { Longitud del pelo }<8.0 \mathrm{~mm} \text {, siempre con escama coronal } \\
\text { divaricada o divergente (Molossidae) }\end{array}$ & 5 \\
\hline $4^{\prime}$ & $\begin{array}{l}\text { Longitud del pelo }>8.1 \mathrm{~mm} \text {, si es menor, entonces la escama } \\
\text { coronal alternada }\end{array}$ & 12 \\
\hline 5 & $\begin{array}{l}\text { Dimensión de la escama, intermedia (IS entre } 0.95 \text { y } 1.10 \text { ), con } \\
\text { bordes denticulados (Tomopetinae) }\end{array}$ & Tomopeas ravus \\
\hline $5^{\prime}$ & $\begin{array}{l}\text { Dimensión de la escama, ancha (IS }<0.94 \text { ), con bordes } \\
\text { dentados (Molossinae) }\end{array}$ & 6 \\
\hline 6 & Escama coronal divergente & 7 \\
\hline $6^{\prime}$ & Escama coronal divaricada & 9 \\
\hline 7 & Pelo con dos bandas de color & 8 \\
\hline $7^{\prime}$ & Pelo con tres bandas de color & Promops davisoni \\
\hline 8 & Longitud del pelo $<6.3 \mathrm{~mm} ;$ AmaxE $<13 \mu \mathrm{m}$ & Molossus molossus \\
\hline $8^{\prime}$ & Longitud del pelo $>6.4 \mathrm{~mm}$; AmaxE $>13.1 \mu \mathrm{m}$ & Tadarida brasiliensis \\
\hline 9 & IS $>0.8$ & \\
\hline $9^{\prime}$ & IS $<0.79$ & 10 \\
\hline 10 & Índice de Ancho de Escama $(\mathrm{WI})>2.16$ & $\begin{array}{l}\text { Mormopterus } \\
\text { kalinowskii }\end{array}$ \\
\hline $10^{\prime}$ & Índice de Ancho de Escama (WI) $<2.15$ & 11 \\
\hline 11 & Borde de la escama con dientes pequeños, $<$ LE $/ 4$ & $\begin{array}{l}\text { Nyctinomops } \\
\text { aurispinosus }\end{array}$ \\
\hline $11^{\prime}$ & Borde de la escama con dientes grandes, $>$ LE $/ 4$ & $\begin{array}{l}\text { Nyctinomops } \\
\text { laticaudatus }\end{array}$ \\
\hline 12 & WI $>1.69$, escamas con borde aserrado (Furipteridae) & $\begin{array}{l}\text { Amorphochilus } \\
\text { schnablii }\end{array}$ \\
\hline $12^{\prime}$ & $\mathrm{WI}<1.68$, escamas con borde no aserrado (Phyllostomidae) & 13 \\
\hline 13 & $\begin{array}{l}\text { Escama coronal divergente; con bordes hendidos } \\
\text { (Desmodontinae) }\end{array}$ & Desmodus rotundus \\
\hline $13^{\prime}$ & Escama coronal alternada; con bordes simples o denticulados & 14 \\
\hline 14 & $\begin{array}{l}\text { Pelo con tres bandas de color, borde de las escamas } \\
\text { denticulado }\end{array}$ & Sturnira bogotensis \\
\hline $14^{\prime}$ & $\begin{array}{l}\text { Pelo con dos bandas de color, borde de las escamas lisas, } \\
\text { simples (Glossophaginae) }\end{array}$ & 15 \\
\hline 15 & Longitud del pelo $<7.6 \mathrm{~mm}$; IS $>1.20$ & Glossophaga soricina \\
\hline $15^{\prime}$ & Longitud del pelo $>7.7 \mathrm{~mm}$; IS $<1.19$ ) & Platalina genovensium \\
\hline
\end{tabular}


en $\mathrm{V}$, mientras que van Staaden y Jones (1997) indican que los murciélagos tienen escamas coronales de tipo divergente o divaricada. Tadarida brasiliensis presentó escamas más divergentes que Nyctinomops y las escamas en Nyctinomops aurispinosus son menos divergentes que las de otros miembros de ese género. Amman et al. (2002) concluyeron que las escamas tipo divaricada y divergente son características de esta familia, con excepción de los murciélagos $N$. macrotis y T. brasiliensis que presentan escamas coronales dentadas; Pierallini et al. (2004) describen escamas coronales divaricadas, Baca y Sánchez-Cordero (2004) mencionan que las escamas coronales dentadas son propias de T. brasiliensis, Schaetz et al. (2009) observaron escamas coronales divaricadas con más de seis dientes visibles, Debelica y Thies (2009) mencionan que el género Myotis tiene escamas coronales simples y que $T$. brasiliensis tiene escamas coronales dentadas al igual que el género Nyctinomops. De otra parte, Sessions et al. (2014) coindice con Debelica y Thies (2009) en la descripción de los géneros Nyctinomops y Tadarida. En el presente estudio se concuerda con los autores que describen las escamas coronales como divaricadas o divergentes y denticuladas o dentadas. Esto se comprueba en la subfamilia Tomopeatinae (Tomopeas ravus) con escamas divaricadas denticuladas y en la subfamilia Molossinae (Mormopterus kalinowskii, Nyctinimops aurispinosus, $N$. laticaudatus, $N$. macrotis y Tadarida brasiliensis) con escamas divaricadas dentadas y escamas divergentes dentadas (Molossus molossus y Promops davisoni).

- En la familia Vespertilionidae se encontró que las escamas son de tipo hastada asimétrica. Benedict (1957) observó escamas coronales hastadas, donde las escamas distales pueden ser sinuadas, enteras o lobadas, pudiendo diferenciarse algunos géneros. Albayrak y Coban (1997) describen las escamas del género Myotis con forma de vaso, y las del género Tadarida con forma de espiga; Amman et al. (2002) indican que los murciélagos vespertilionidos presentan formas de escama que van desde coronal lobado amplio a coronal hastado, pero nunca corona dentada; Pierallini et al. (2004) observaron escamas coronales divergentes; y Schaetz et al. (2009) describen escamas imbricadas con forma de lengüeta y escamas imbricadas truncas. Los resultados de este estudio concuerdan con Benedict (1957) y Schaetz et al. (2009) ya que en esta familia (Eptesicus montanus, Myotis atacamensis y $M$. cf. oxyotus) se observaron las escamas coronales de tipo alternada asimétrica.

Dichas variaciones en las descripciones son aparentemente producto de las limitaciones tecnológicas de la época; por consiguiente se espera que los resultados aquí presentados sirvan de un marco de referencia contemporáneo.

A nivel ecológico y anatómico se han planteado algunas hipótesis para explicar las modificaciones de la morfología de la cutícula en los pelos de murciélagos. Así, Howell y Hodking (1976) mencionan que los murciélagos polinizadores presentan escamas muy divergentes y divididas que les permitiría colectar una mayor cantidad de polen de plantas, mientras que los murciélagos no asociados a flores presentan escamas más apretadas. Así mismo, Meyer et al. (1995) sostienen que ciertas formas de cutícula facilitan el vuelo debido a que generan una menor turbulencia. No obstante, es necesario más estudios que permitan explicar la variada morfología cuticular y la función que cumplen en las diversas familias de murciélagos que ocurren en Perú. 


\section{Conclusión}

- Se registraron cinco tipos de patrones cuticulares coronales y seis configuraciones del borde de las escamas, los cuales junto con datos morfométricos permiten diferenciar los especímenes hasta el nivel de especie.

- Se documenta, por primera vez, una descripción de la cutícula de pelos de guardia para Glossophaga valens, Platalina genovensium, Sturnira bogotensis, Amorphochilus schnablii, Tomopeas ravus, Molossus molossus, Mormopterus kalinowskii, Nyctinomops aurispinosus, Nyctinomops laticaudatus, Nyctinomops macrotis, Promops davisoni, Tadarida brasiliensis, Eptesicus montanus, Myotis atacamensis y Myotis cf. oxyotus.

\section{Agradecimientos}

Se agradece a los investigadores asociados y voluntarios de la Colección Científica del Museo de Historia Natural de la Universidad Nacional de SanAgustín de Arequipa (MUSA), en especial a los miembros del área de mastozoología por el apoyo con la preparación de muestras permanentes. Asimismo, a los miembros del Área de Biología Forense de la Oficina de Criminalística - Sede Arequipa, de la Policía Nacional del Perú. El estudio se realizó con financiamiento de la Universidad Nacional de San Agustín de Arequipa (UNSA), mediante Contrato de Subvención N. ${ }^{\circ}$ 14-2016-UNSA.

\section{Literatura Citada}

1. Amman BR, Robert DO, Bradley RD. 2002. Utility of hair structure for taxonomic discrimination in bats, with an example from the bats of Colorado. Occasional Paper Museum of Texas Tech Univ 216: 1-14. doi: 10.5962/bhl.title. 156835

2. Albayrak I, Coban N. 1997. Hair morphology of some mammalian species in Turkey. Comm FacSci Ankara Series C 15:21-39
3. Arita H, Aranda M. 1987. Técnicas para el estudio y clasificación de los pelos. Cuadernos de Divulgación INIREB 32: 1-21.

4. Baca II, Sánchez-Cordero V. 2004. Catálogo de pelos de guardia dorsal en mamíferos del estado de Oaxaca, México. An Inst Biol 75: 383-437.

5. Benedict FA. 1957. Hair structure as a generic character in bats. Quat Rev Biol 59: $285-548$

6. Brown MT, Wicker LR. 2000. Discriminant analysis. In: Tinsley HEA, Brown SD (eds). Handbook of applied multivariate statistics and mathematical modeling. California, USA: Academic Press. p 209-235.

7. Debelica A, Thies ML. 2009. Atlas and key to the hair of terrestrial Texas mammals. Museum Texas Tech Univ 55: 1-102. doi: 10.5962/bhl.title.142652

8. Cabrera A. 1922. Manual de mastozoología. Barcelona, Madrid: Compañía Anónima de Librería, Publicaciones y Ediciones.. $440 \mathrm{p}$.

9. Fasola L, Bello M, Guichón ML. 2005. Uso de trampas de pelo y caracterización de los pelos de la ardilla de vientre rojo Callosciurus erythraeus. Mastozool Neotrop 12: 9-17.

10. Fernández G, Rossi S. 1998. Medullar type and cuticular scale patterns of hairs of rodents and small marsupials from the Monte Scrubland (San Luis province, Argentina). Mastozoología Neotrop 5: 109-116.

11. Hammer 0. 2013. PAST: paleontological statistics software package for education and data analysis v. 3.0. Reference manual. 275 p.

12. Howell DJ, Hodgkin N. 1976. Feeding adaptations in the hairs and tongues of nectar feeding bats. J Morphol. doi: https://doi.org/10.1002/jmor.1051480305

13. Kshirsagar SV, Singh B, Fulari SP. 2009. Comparative study of human and animal hair in relation with diameter and medullary index. Ind J Forensic Med Pathol 2: 105-108. 
14. Kumar M, Priya YS, Mathur V, Kumar H, Elangovan V. 2014. Scanning electron microscopy study of guard hair of three Indian pteropodid bats. Mammalia 78: 533-537. doi: 10.1515/ mammalia-2013-0103

15. Málaga BA, Díaz DR, Arias $S$, Medina CE. 2020. Una nueva especie de Lasiurus (Chiroptera: Vespertilionidae) del suroeste de Perú. Rev Mex Biodivers 91: e913096. doi: 10.22201/ib.20078706e.2020.91.3096

16. Martin RE, Pine RH, Blase AF. 2001. A manual of mammalogy: with keys to families of the world. $3^{\text {rd }}$ ed. USA: Waveland Press. 352 p.

17. Mathiak HA. 1938. A key to hairs of the mammals of southern Michigan. J Wildlife Manage 2: 251-268. doi: 10.2307/ 3795673

18. Medina CE, Díaz DR, Téllez B, Medina YK, López E. 2018. Second record of Eumops chiribaya (Chiroptera: Molossidae) in Perú. Biodiversitas 19: 1979-1984. doi: 10.13057/biodiv/ d190601

19. Meyer W, Seger H, Hulmann G 1995. Remarks on specific adaptive scale structure of the hair cuticle in some European bats. Eur J Morphol 33: 509-513.

20. Monroy-Vilchis O, García C, Rubio R, Hernández A, Medina J, Aguilera U, Ortiz $A$. 2005. Variación intraespecífica e individual de los pelos de mamíferos del estado de México: implicaciones en la identificación interespecífica. Ciencia Ergo Sum 12: 264-270.

21. Moore DW, Braun JK. 1983. Keys to the hairs of the families Soricidae, Vespertilionidae, and Muridae within Tennessee. J Tenn Acad Sci 58: 40-43

22. Nason ES. 1948. Morphology of hair of Eastern North American bats. Am Midl Nat 39: 345-361. doi: 10.2307/ 2421589
23. Pari A, Pino KS, Medina CE, Zeballos H, López E. 2015. Murciélagos de Arequipa. Historia natural y conservación. Colección Científica. Arequipa, Perú: Museo de Historia Natural de la Universidad Nacional de San Agustín (MUSA). $181 \mathrm{p}$.

24. Pierallini R, Keller A, Moretti M. 2004. Chiave di determinazione dei Chirotteri (Mammalia) della Svizzera attraverso l'osservazione al microscopio ottico della struttura dei peli. Rev Suisse Zool 111: 381-394. doi: 10.5962/bhl.part.80244

25. Quadros J, Monteiro-Filho ELA. 1998. Effects of digestion, putrefaction and taxidermy processes on Didelphis albiventris hair morphology. J Zool 224: 331-334. doi: 10.1111/j.1469-7998.1998.tb00037.x

26. Quadros J, De A Monteiro-Filho EL. 2006. Coleta e preparação de pêlos de mamíferos para identificação em microscopia óptica. Rev Bras Zool 23: 274-278. doi: 10.1590/S0101-81752006000100022

27. Schaetz BA, Kurta A, RodríguezDsurán A, Münzer OM, Foster R. 2009. identification of bats in Puerto Rico using the scanning electron microscope to examine body hairs. Caribb J Sci 45: 125-130. doi: 10.18475/cjos.v45i1.a1

28. Sessions BD, Nielson CE, Sowa JM, Hess WM, Skidmore W, Carmack BA. 2014. Is bat hair morphology exceptional? Vespertilio 17: 171-183

29. Teerink BJ. 1991. Hair of west European mammals: atlas and identification. Cambridge, UK: Cambridge University Press. 224 p.

30. Van Staaden M, Jones Jr JK. 1997. Comparative morphology of dorsal hair of New World bats of the family Molossidae. In: Arroyo-Cabrales J, Polaco OJ (eds). Homenaje al profesor Ticul Álvarez. Colección Científica. México DF: Instituto Nacional de Antropología e Historia. p 373-391. 